\title{
Rational incorporation of missing linker defects within metal-organic frameworks generates highly active electrocatalytic sites
}

\author{
Nina Heidary ${ }^{1}$, Daniel Chartrand ${ }^{1}$, and Nikolay Kornienko ${ }^{1 *}$ \\ ${ }^{1}$ Department of Chemistry, Université de Montréal, 1375 Avenue Thérèse-Lavoie-Roux, Montréal, QC \\ H2V 0B3, Canada. \\ *E-mail: nikolay.kornienko@umontreal.ca
}

\begin{abstract}
:
The allure of metal-organic frameworks (MOFs) in heterogeneous electrocatalysis is that catalytically active sites may be designed a priori with an unparalleled degree of control. An emerging strategy to generate coordinatively-unsaturated active sites is through the use of organic linkers that lack a functional group that would usually bind with the metal node. To execute this strategy, we synthesize a model MOF, Ni-MOF-74 and incorporate a fraction of 2-hydroxyterephthalic acid in place of 2,5-dihydroxyterephthalic acid. The defective MOF, Ni74D, is evaluated vs. the nominally defect-free Ni74 MOF with a host of $e x$ situ and in situ spectroscopic and electroanalytical techniques, using the oxidation of hydroxymethylfurtural (HMF) as a model reaction. The data indicates that Ni74D features a set of 4-coordinate $\mathrm{Ni}^{-\mathrm{O}_{4}}$ sites that exhibit unique vibrational signatures, redox potentials, binding motifs to HMF, and consequently superior electrocatalytic activity relative to the original Ni74 MOF, being able to convert HMF to the desired 2,5furandicarboxylic acid at $95 \%$ yield and $80 \%$ Faradaic efficiency. The strategy put forth to rationally design coordinatively-unsaturated electrocatalytic sites and the methodology put forth in investigating their behavior stand to bolster the understanding and growth of the field.
\end{abstract}




\section{Introduction:}

The utilization of metal and covalent organic frameworks (MOFs and COFs) in electrochemical systems is increasingly attracting interest because of the unique capabilities of these structures. ${ }^{1-5}$ Through judicious selection of the organic linker and metal nodes, the porosity, internal chemistry, conductivity, active site physical and electronic structure can all be chosen a priori to generate the ideal catalyst. This degree of control is unmatched by conventional heterogeneous and molecular materials and thus opens up unique parameter spaces to explore.

In regard to the tuning of the active site several strategies have been explored in recent years. Molecular catalysts serving as the organic linker within MOFs and COFs gave rise to several highly functional catalytic systems for the hydrogen evolution reaction (HER), ${ }^{6-7} \mathrm{CO}_{2}$ reduction reaction $\left(\mathrm{CO}_{2} \mathrm{R}\right){ }^{8-}$ 12 and oxygen reduction reaction (ORR). ${ }^{13}$ The activity of the molecular active could be further tuned through electron withdrawing functional groups grafted within the COF structures. ${ }^{14}$ The metal nodes of MOFs could also serve as active sites for the HER ${ }^{15}$ and the oxygen evolution reaction (OER), ${ }^{16-19}$ and the activity these can be augmented through lattice strain ${ }^{20}$ or modulation of the linker electronic structure. Finally, the reaction environment within MOFs and related systems can be tuned to impart secondary coordination sphere effects in an enzyme-inspired fashion. ${ }^{21}$ This has recently been exemplified through the grafting of pyridine units that stabilize the $\mathrm{CO}_{2}{ }^{-}$intermediate on a Co-protoporphyrin active site en route to the reduction of $\mathrm{CO}_{2}$ to $\mathrm{CO}$ in a metal-organic layer. ${ }^{22}$

One strategy recently established in the MOF community is the use of organic linkers that are missing functional groups that bind to the metal nodes called missing linkers. Successful incorporation of such missing linkers consequently results in a fraction of the metal nodes that are coordinatively unsaturated. Missing linker defect sites have primarily been applied in gas sorption, ${ }^{23}$ chemical separations, ${ }^{24}$ liquid phase catalytic transformations ${ }^{25}$ and proton conductivity. ${ }^{26}$

In the context of electrocatalysis, these unsaturated sites have the potential to be highly active and their rational design and incorporation within an electrocatalytic MOF stands to augment its activity. This has recently been demonstrated for the OER, ${ }^{27}$ in which unsaturated Co nodes exhibited a favorable electronic structure to facilitate water oxidation. As this strategy is rather nascent, neither a complete understanding of the resultant undercoordinated sites nor the methodology to evaluated them is yet established. Against this backdrop, we moved to explore a missing linker MOF with a unique combination of spectroscopic and electroanalytical techniques using the aqueous electrooxidation of hydroxymethylfurfural (HMF) ${ }^{28}$ as a model reaction and important challenge in the emerging area of electrochemical biomass valorization, which carries the potential to use renewable electricity to generate, from abundant raw ingredients, many of the fuels and chemicals that power society. ${ }^{29}$

We chose Ni-MOF-74 as our model system. This MOF, first synthesized in $2005,{ }^{30}$ features chains of $\mathrm{Ni}^{2+}$ linked together by 2,5-hydroxyterepthalate to give rise to cylindrical pores (Fig. 1a). The Ni sites typically feature 6-fold coordination but upon solvent exchange and removal can become 5-coordinated, leaving open an uncoordinated site that has previously proven to be beneficial for $\mathrm{CO}_{2}$ sorption $(\mathrm{Mg}, \mathrm{Ni}$, Co, Zn-MOF-74), ${ }^{31}$ toxic gas adsorption (Mg, Ni, Co, Zn-MOF-74), ${ }^{32}$ and $\mathrm{NO}_{\mathrm{x}}$ reduction(Mn, Co-MOF74), ${ }^{33}$ It is important to note that both, the carboxylate and alcohol groups of the 2,5-hydroxyterepthalate linker bind with the Ni chains. Therefore, the partial incorporation of 2-hydroxyterepthalate in place of the original linker would result in 4-coordinate $\mathrm{Ni}$ centers what we hypothesized to be exceptionally active in an electrochemical context (Fig. 1b-d). As a recent reference point, the coordinatively unsaturated Ni-O centers at the edges of $\mathrm{NiOH}_{2}$ nanoribbons were found to be highly efficient for electrocatalytic water and methanol oxidation. ${ }^{34-35}$ In the last year, transition metal sites in MOFs explored as active sites for HMF 
oxidation. ${ }^{36-37}$ We moved to systematically study the structure and activity of these missing linker defect $\mathrm{NiO}_{4}$ sites within Ni-MOF-74 with the aim of generating an in depth understanding of how they function in an electrocatalytic context and by doing so, extract out valuable design principles that can be generalized across a wide array of systems.
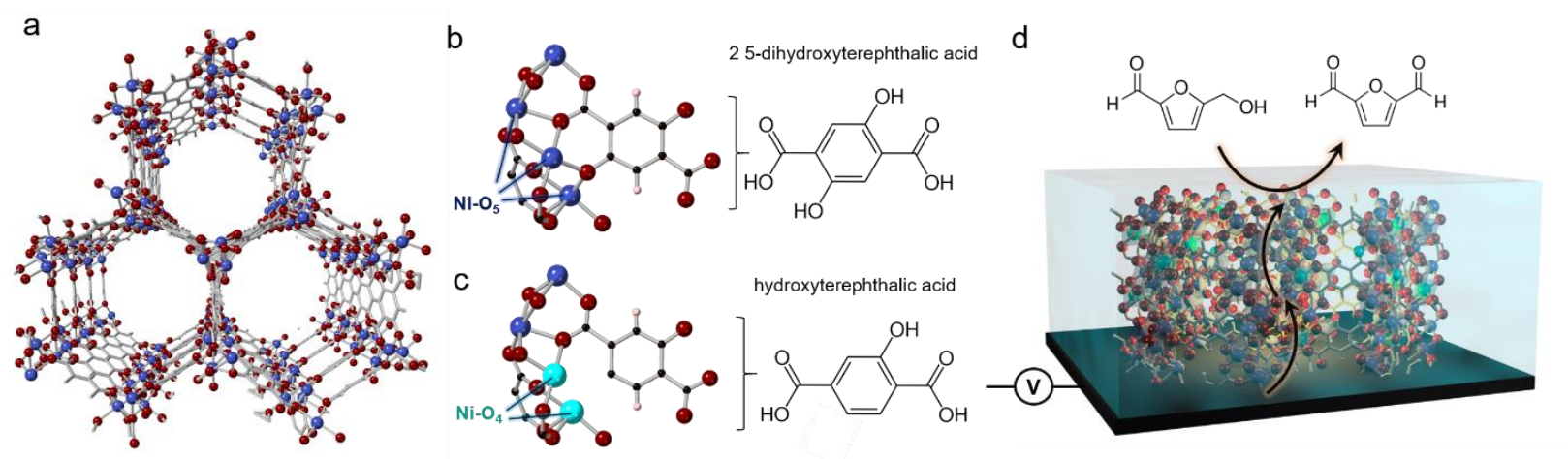

Figure 1: Ni-MOF 74 contains cylinder shaped pores and chains of 5-coordinate Ni oxide units (a). The 2,5-dihydroxyterepthalic acid organic linker, will bind through both the carboxylate and alcohol units (b) and replacing it with 2-hydroxyterepthalic acid will result in defect sites of 4-coordinate Ni sites (c). We studied the effects of these defect sites with electrochemical and spectroscopic techniques, using the oxidation of HMF as a model reaction (d).

\section{Results and Discussion:}

We proceeded to synthesize our target materials with a simple one-pot solution phase approach, adapting a previously established recipe in which missing linker defects were generated in Co-MOF-74. ${ }^{38}$ The nominally defect-free MOF, Ni74, was synthesized with only 2,5-dihydroxyterepthalic acid while the defect-containing MOF, Ni74D, was synthesized using a 3:1 ratio of 2,5-dihydroxyterepthalic acid: 2hydroxyterepthalic acid (Fig. 2a). After synthesis, solvent exchange and evacuation, the two MOFs were first characterized with ex situ methods. The x-ray diffraction (XRD) spectrum for both MOFs was wellmatched to what was expected for MOF-74 (Fig. 2b). The main difference noticeable in the spectra was that Ni74D features slightly broader peaks which were shifted to higher 2-theta values, indicating a lower degree of crystallinity and a smaller unit cell, both expected observations for defect-containing MOFs. ${ }^{39-41}$ Additional reflections pointing to a periodicity in the defects was not observed in the Ni74D XRD spectrum so we therefore assume the defects to be distributed without any particular ordering (Fig. S1).

Scanning electron microscopy (SEM) revealed the two MOFs to consist of particles on the order of 2-5 micrometers in diameter without any particular shape (Fig. 2c,d). However, noticeable differences were observed in the infrared (IR) spectrum. The IR absorption bands were broader for (Fig. S2), indicating a lower degree of homogeneity as already evidenced through XRD measurements. However, the most interesting aspect in the IR spectra was the band attributed to the Ni-O vibrations centered around 595-598 $\mathrm{cm}^{-1}$ (Fig. 2e). Previous studies of nickel oxides and hydroxides have recorded characteristic bands in this region. ${ }^{19,}{ }^{42-43}$ For Ni74, the spectra could be well-fit with two peaks at 595 and $580 \mathrm{~cm}^{-1}$. However, the broader band of Ni74D had to be fit with a combination of 3 peaks, at 595, 579 and $609 \mathrm{~cm}^{-1}$. This indicates 
that there is a greater distribution of coordination environments of the Ni centers within Ni74D, as expected for a defect containing MOF.

a
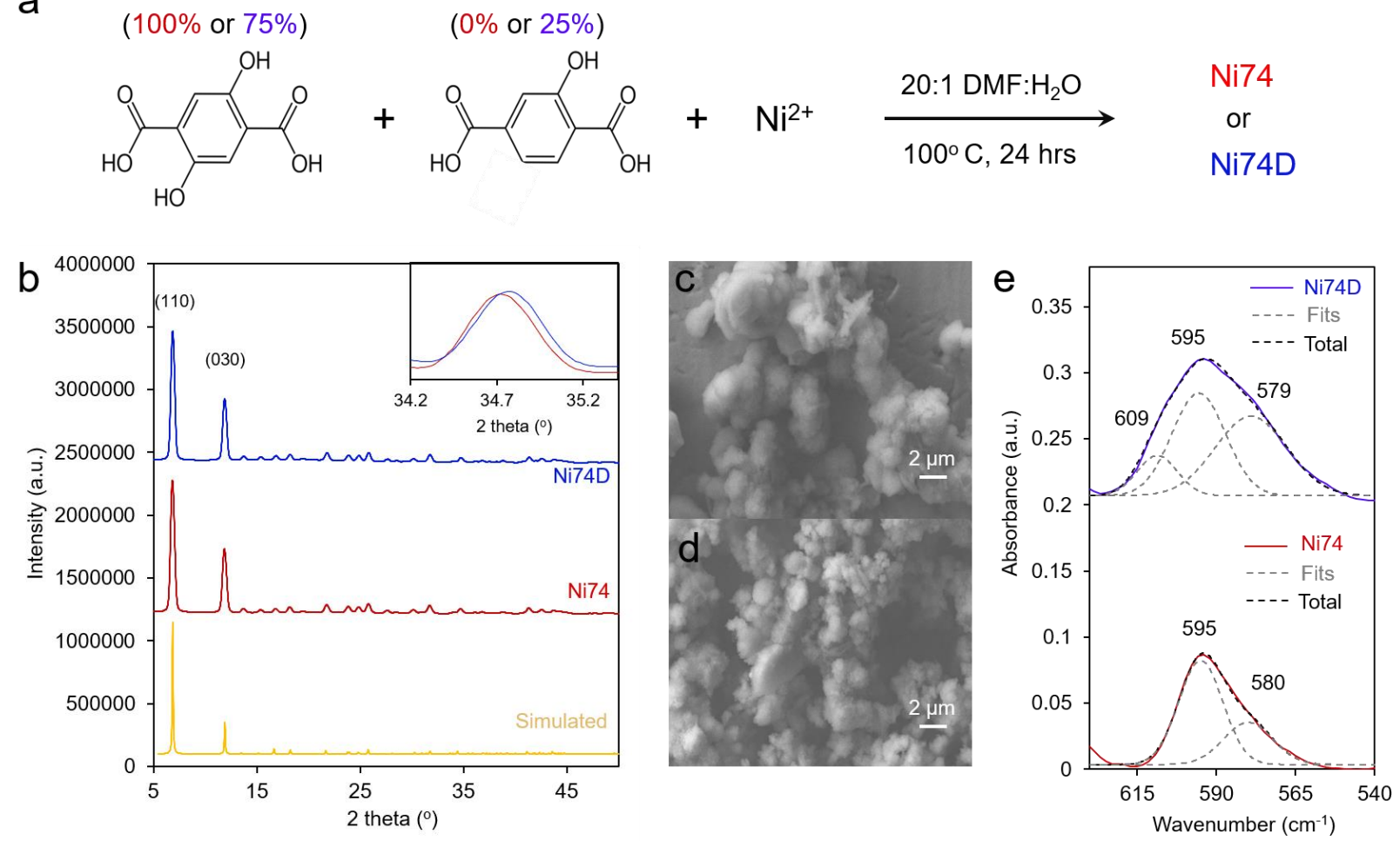

Figure 2: The synthesis of the Ni74 and Ni74D MOFs entailed the use of only dihydroxy and a combination of mono and dihydroxyterepthalic acid linkers (a). Both MOFs exhibited the same XRD spectrum, through Ni74D featured a lower degree of crystallinity (b). Both MOFs were micron sized (c, d), though the Ni-O absorption band of Ni74D was broader and composed of three distinct components (e).

As spectroscopic measurements can be exceptionally instrumental in understanding catalystreactant interactions, ${ }^{44}$ we therefore moved to study the MOFs, and in particular their interactions with the electrolyte and HMF with IR spectroscopy in an ATR configuration (Fig. 3a). The first area of interest was the low-frequency region in which the $\mathrm{Ni}-\mathrm{O}$ vibrations could be found. Upon immersion in $10 \mathrm{mM} \mathrm{KOH}$ electrolyte, the Ni-O bands red-shifted for both Ni74 and Ni74D (Fig. 3b), as expected due to differences in environment and presence of hydrogen bonding. Interestingly, a new peak appeared at $554 \mathrm{~cm}^{-1}$ that we tentatively attribute to adsorbed $\mathrm{OH}$ onto the open metal sites. Bands attributed to Ni-O-H vibrations on $\mathrm{NiOH}_{2}$ been previously observed at this position. ${ }^{19,43,45}$ The new band was much stronger on Ni74D as this material had more open metal sites onto which OH could adsorb. With HMF in the solution, the band at $554 \mathrm{~cm}^{-1}$ decreased slightly in intensity for both systems. This is tentatively interpreted as a fraction of the $\mathrm{OH}$ becoming displaced by HMF coordinated to the Ni centers.

The interaction of the HMF with the Ni centers of the two materials was subsequently probed in the high-frequency region in which the $\mathrm{C}=\mathrm{C}$ and $\mathrm{C}=\mathrm{O}$ stretching vibrations exist. After carefully subtracting out the spectra of the hydrated MOF, we were able to isolate the spectral features of the HMF (Fig. 3c). 
The bands at 1660 and $1524 \mathrm{~cm}^{-1}$ correspond to HMF in the solution that was not interacting with the MOF. However, a new band arose centered $1548-1558 \mathrm{~cm}^{-1}$ in the presence of the MOFs. The band was redshifted and broader for Ni74D. The broadening indicates that there is likely a distribution of different interactions present as Ni74D features both the 4-coordinate and 5-coordinate $\mathrm{Ni}$-O centers that can interact with HMF. We attribute this new band to the $\mathrm{C}=\mathrm{O}$ stretch as the $\mathrm{C}=\mathrm{O}$ bond of the aldehyde group is weaker upon interacting with the $\mathrm{Ni}$ centers and thus the band is re-shifted. Within the same line of reasoning, the redshift of the coordinated aldehyde band would also provide evidence for a stronger interaction of HMF with the Ni74D. New emergent bands in this spectral region have been observed before for HMF interacting with $\mathrm{Au}, \mathrm{NiOH}_{2} / \mathrm{NiOOH}$ and $\mathrm{CoO}_{x}$ surfaces. ${ }^{46-47}$

a

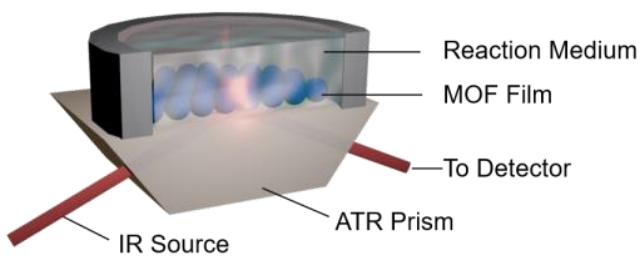

$\mathrm{b}$
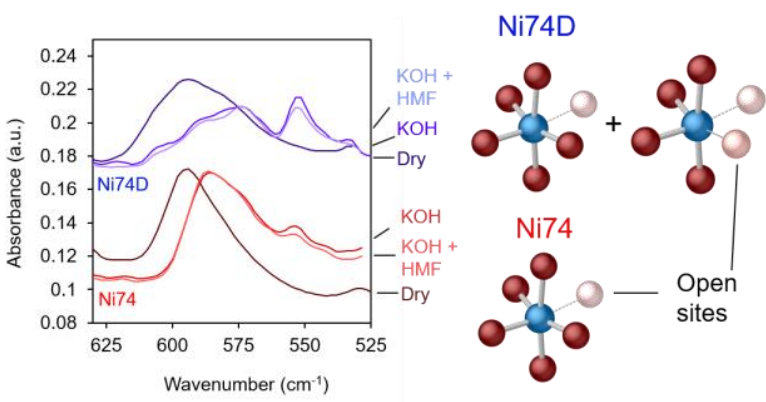

C

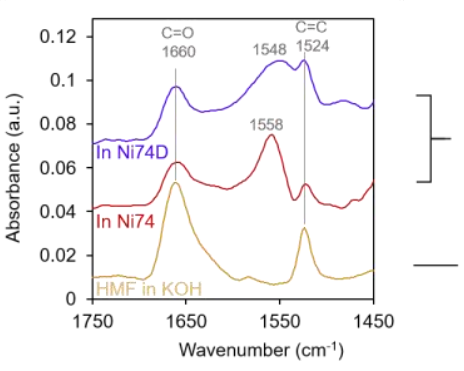

$\mathrm{Ni}$

Coordinated HMF

Ni<smiles>C1CCC2=C(C1)CCO2</smiles>

Solubilized HMF

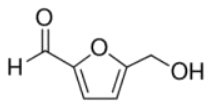

Figure 3: An ATR configuration was used to probe the MOF-HMF interactions (a). The low wavenumber spectra reveal that $\mathrm{Ni74D}$ has more open coordination sites in which $\mathrm{OH}$ can adsorb, which is then partially displaced by HMF (b). The HMF-Ni interactions can also be visualized in the high wavenumber region in which a new band arises, stemming from the interaction of the HMF aldehyde group with the Ni centers of the MOFs (c).

We finally set out to study the catalytic effect that the defect sites imparted onto Ni74D with an array of electrochemical measurements in a $10 \mathrm{mM} \mathrm{KOH}$ electrolyte. Briefly, electrodes were constructed by making a catalyst ink by sonicating together carbon nanotubes, nafion, and the desired material in a 3:1 vol:vol ethanol:water solution $(10 \mathrm{mg} / \mathrm{ml})$. This ink was drop cast onto a carbon paper substrate to attain a 
final MOF loading of $1 \mathrm{mg} / \mathrm{cm}^{2}$ and allowed to dry in ambient conditions. Because of the relatively low conductivity of both MOFs (Fig. S3), differential-pulse voltammetry (DPV) was used to prove the Ni(II/III) redox potential. The redox wave of Ni74D was broadened and shifted to more negative potentials relative to Ni74, indicating that both, a distribution of redox environments existed (e.g. 4- and 5-coordinated Ni-O) and that the 4-coordinate Ni centers were easier to oxidize (Fig. 4a). The interactions of the Ni centers with HMF were complementarily probed with open circuit voltage measurements (Fig. 4b). When $10 \mathrm{mM} \mathrm{HMF}$ was injected into the electrolyte solution, the potential for the Ni74 MOF shifted in the negative direction more than that of Ni74D. This signifies that, on average, the 5-coordinate Ni-O centers accept more charge from $H M F$ in their interactions with it.

In the cyclic voltammetry (CV) measurements, Ni74 featured only a small increase of current between 0.9 and $1.4 \mathrm{~V}$. In contrast, Ni74D exhibited catalytic current for HMF oxidation even before the $\mathrm{Ni}$ (II/III) redox potential. Differences in the catalytic activity of the two systems were also visible in the different Tafel slopes for HMF oxidation ( $160 \mathrm{mV} / \mathrm{dec}$ for Ni74D, $300 \mathrm{mV} / \mathrm{dec}$ for Ni74), taken near the onset of the catalytic current, just past the $\mathrm{Ni}$ (II/III) redox potential in a rotating disk electrode setup before mass transport limitations came into effect. After a long-term $20 \mathrm{hr}$ electrolysis of $10 \mathrm{mM} \mathrm{HMF}$, the reactant was completely converted to 2,5-furandicarboxylic acid (FDCA) at 95\% yield as both the aldehyde and alcohol groups of HMF were completely oxidized to the carboxylic acid (Fig. 4e). FDCA is the highestvalue product from HMF oxidation and is an important precursor to polymers. NMR spectra taken at short measurement times showed the presence of furan-2,5-dicarbaldehyde (DFF) and 5-formyl-2furancarboxylic acid (FFCA), indicating that the reaction proceeds through the oxidation of the alcohol group of the HMF first, rather than the aldehyde group (Fig. S4). The turnover number, determined by dividing the molecules of FDCA detected by the quantity of electrochemically addressable Ni sites, was determined to be 1520 and the Faradaic efficiency for FDCA formation to be $80 \%$, with water oxidation attributed to the rest of the charge passed. This performance is exceptional, especially in $\mathrm{pH} 12$. In comparison, $\mathrm{Au}$ and Ni electrodes only showed minimal ( $<1 \%$ conversion) FDCA production. The stability of the system was evaluated with a combination of SEM and XRD measurements. SEM images showed that the morphology of the Ni74D is visibly similar after a $24 \mathrm{hr}$ catalytic run at $1.4 \mathrm{~V}$ (Fig. S5). Similarly, the expected diffraction pattern could still be detected after catalysis (Fig. S6). 

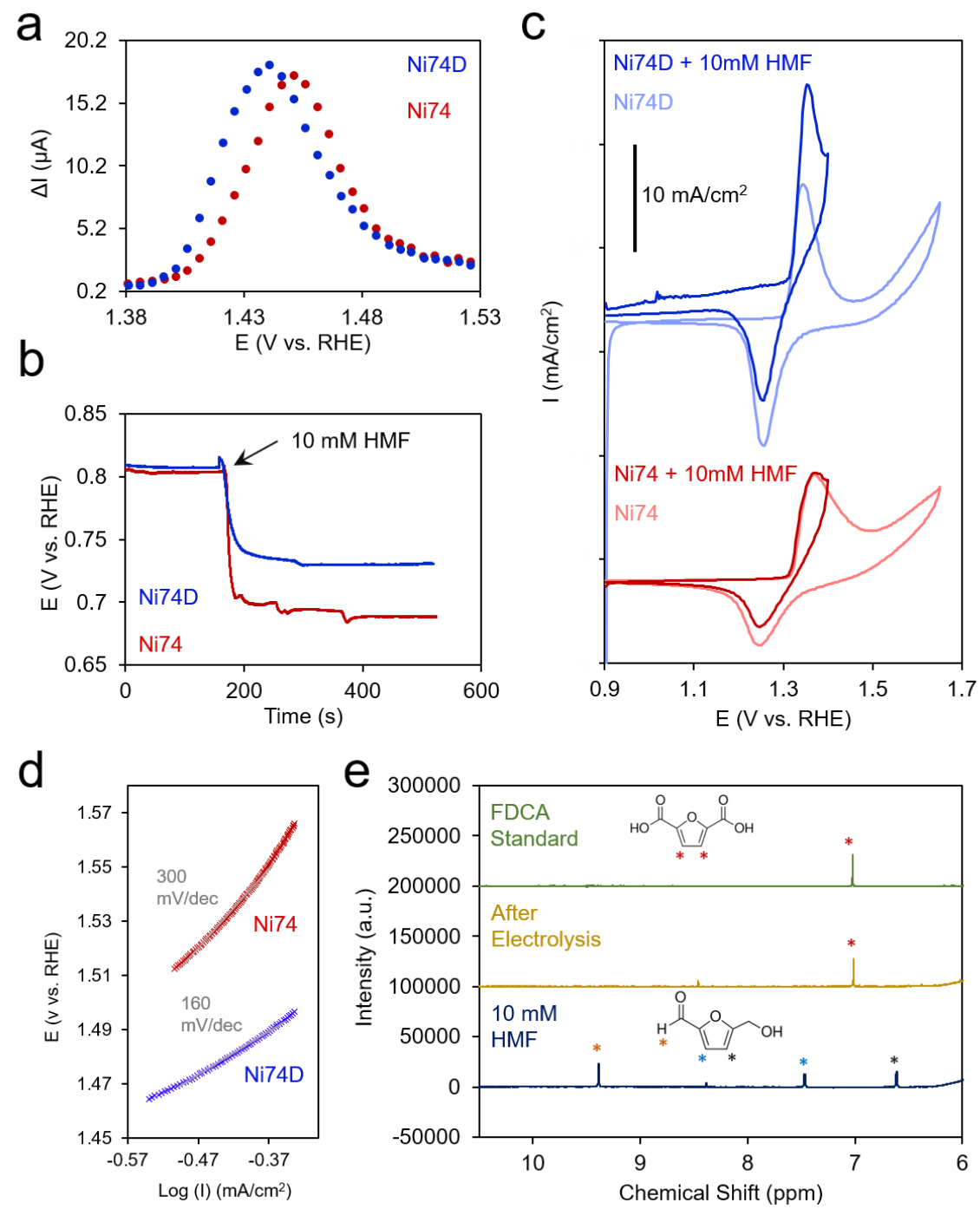

Figure 4: DPV measurements illustrate differences in the redox behavior of Ni74D and Ni74 (a). Their differing interaction with HMF is also evidenced through open circuit potential measurements (b). Ni74D features catalytic current even prior to the $\mathrm{Ni}(\mathrm{II} / \mathrm{III})$ redox wave, at which its catalytic activity further increases (c). Catalytic differences are also evident by differences in Tafel slopes in a rotating disk configuration (d). HMF could be completely converted to FDCA after extended electrolysis shown in NMR spectra (e).

From the sum of the spectroscopic and electrochemical data, there are several factors that we can speculate on that lead to the enhanced performance of Ni74D for HMF oxidation relative to Ni74. Taking a molecular view on the active sites, the resultant 4-coordinate Ni sites can exhibit several possible HMF binding motifs. A single binding interaction to the $\mathrm{Ni}$ site may be possible (Fig. 5a), as well as a simultaneous interaction between two Ni sites (Fig. 5b) which are typically 3.1 A apart. Note, this bidentate type of coordination does not need to necessarily coordinate to the same atom on HMF. Furthermore, lateral interactions between HMF adsorbed on one site and an interacting species adsorbed on an adjacent site may influence the reaction (Fig. 5c). For example, species such as $* \mathrm{OH}$ or $* \mathrm{O}$ that are water oxidation intermediates have been shown to facilitate alcohol oxidation reactions. ${ }^{48-50}$ Finally, both HMF and, for 
example, ${ }^{*} \mathrm{OH}$ could be adsorbed on the same 4-coordinate Ni site and interact with each other (Fig. 5d). While the binding motif in Fig. 5d is only possible with the 4-coordinate Ni sites, the interactions in 5a-c, while possible, would all be different be different on Ni74 with 5-coordinate Ni sites due to differences in electronic structure between the two types of Ni. An interesting observation came upon testing Ni74D for oxidation of HMFCA, which possesses only an oxidizable alcohol group, and DFF, having only oxidizable aldehyde groups (Figure S7). HMFCA oxidation occurred prior to the Ni(II/III) redox potential, similar to what was observed for HMF oxidation. In contrast, DFF oxidation required $\mathrm{Ni}$ (III) to be present.

While the electroanalytic and IR experiments granted us an exciting base from which to build upon, there is much work to be done to completely understand this system. X-ray absorption spectroscopy, and in particular the modelling of the extended fine structure (EXAFS) could provide key insights into the local coordination environment of the Ni centers and how it changes under reaction conditions. Similarly, Raman spectroscopy has been key in resolving structure-activity relationships for many heterogeneous catalysts, and in particular, Nickel oxides and could be readily implemented for this system. Finally, density function theory (DFT) simulations may well give insights into favorable reaction pathways and how the interplay of co-adsorbates and multiple adjacent coordinatively unsaturated feeds into the overall performance of these materials.
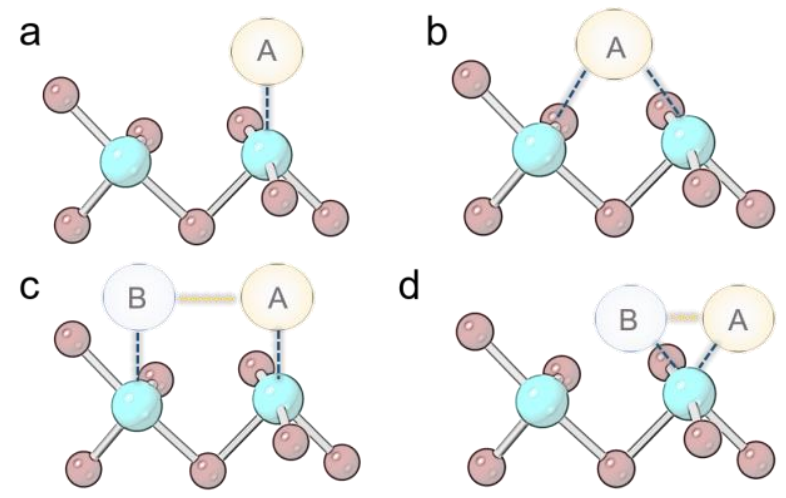

d

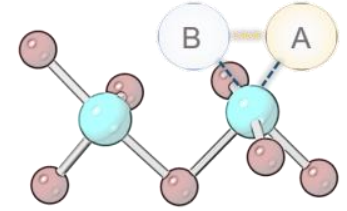

Figure 5: Several types of binding motifs exist between the Ni74D defect sites and HMF (depicted by A spheres). Mono (a) and bidentate (b) HMF binding geometries are possible as well as lateral interactions with co-adsorbed intermediates on neighboring sites (c) and on the same site (d).

\section{Concluding Remarks:}

In sum, we present a combined spectroscopic and electroanalytic study on the catalytic properties of missing linker defect sites in Ni-MOF-74. Using electrochemical HMF oxidation as a model reaction, we show through in situ IR spectroscopy and electrochemical analysis that there are distinct Ni coordination environments in the defect containing MOF that consequently lead to unique interactions with HMF and therefore exceptional electrocatalytic performance. The missing linker strategy and the methods developed in this work to evaluate the resultant defect sites can readily be extended to a variety of different MOF and reactions to fully take advantage of their unique nature. 


\section{Acknowledgements:}

N.K. and N.H. acknowledge NSERC Discovery Grant RGPIN-2019-05927.

\section{References:}

1. Diercks, C. S.; Liu, Y.; Cordova, K. E.; Yaghi, O. M., The role of reticular chemistry in the design of $\mathrm{CO}_{2}$ reduction catalysts. Nat. Mater. 2018, 17 (4), 301-307.

2. Lu, X. F.; Xia, B. Y.; Zang, S.-Q.; Lou, X. W., Metal-Organic Frameworks Based Electrocatalysts for the Oxygen Reduction Reaction. Angew. Chem. Int. Ed. 2020, 59 (12), 4634-4650.

3. Heidary, N.; Harris, T. G. A. A.; Ly, K. H.; Kornienko, N., Artificial photosynthesis with metal and covalent organic frameworks (MOFs and COFs): challenges and prospects in fuel-forming electrocatalysis. Phys. Plant 2019, 166 (1), 460-471.

4. Baumann, A. E.; Burns, D. A.; Liu, B.; Thoi, V. S., Metal-organic framework functionalization and design strategies for advanced electrochemical energy storage devices. Commun. Chem. 2019, 2 (1), 86.

5. Wu, H. B.; Lou, X. W., Metal-organic frameworks and their derived materials for electrochemical energy storage and conversion: Promises and challenges. Sci. Adv. 2017, 3 (12), eaap9252.

6. $\quad$ Roy, S.; Huang, Z.; Bhunia, A.; Castner, A.; Gupta, A. K.; Zou, X.; Ott, S., Electrocatalytic Hydrogen Evolution from a Cobaloxime-Based Metal-Organic Framework Thin Film. J. Am. Chem. Soc. 2019, 141 (40), 15942-15950.

7. Micheroni, D.; Lan, G.; Lin, W., Efficient Electrocatalytic Proton Reduction with Carbon Nanotube-Supported Metal-Organic Frameworks. J. Am. Chem. Soc. 2018, 140 (46), 15591-15595.

8. Matheu, R.; Gutierrez-Puebla, E.; Monge, M. Á.; Diercks, C. S.; Kang, J.; Prévot, M. S.; Pei, X.; Hanikel, N.; Zhang, B.; Yang, P.; Yaghi, O. M., Three-Dimensional Phthalocyanine Metal-Catecholates for High Electrochemical Carbon Dioxide Reduction. J. Am. Chem. Soc. 2019, 141 (43), 17081-17085.

9. Lin, S.; Diercks, C. S.; Zhang, Y.-B.; Kornienko, N.; Nichols, E. M.; Zhao, Y.; Paris, A. R.; Kim, D.; Yang, P.; Yaghi, O. M.; Chang, C. J., Covalent organic frameworks comprising cobalt porphyrins for catalytic CO2 reduction in water. Science 2015, 349 (6253), 1208.

10. Kornienko, N.; Zhao, Y.; Kley, C. S.; Zhu, C.; Kim, D.; Lin, S.; Chang, C. J.; Yaghi, O. M.; Yang, P., Metal-Organic Frameworks for Electrocatalytic Reduction of Carbon Dioxide. J. Am. Chem. Soc. 2015, 137 (44), 14129-14135.

11. Hod, I.; Sampson, M. D.; Deria, P.; Kubiak, C. P.; Farha, O. K.; Hupp, J. T., Fe-Porphyrin-Based Metal-Organic Framework Films as High-Surface Concentration, Heterogeneous Catalysts for Electrochemical Reduction of CO2. ACS Catal. 2015, 5 (11), 6302-6309.

12. Heidary, N.; Morency, M.; Chartrand, D.; Ly, K. H.; Iftimie, R.; Kornienko, N., Electrochemically Triggered Dynamics within a Hybrid Metal-Organic Electrocatalyst. J. Am. Chem. Soc. 2020, 142 (28), 12382-12393.

13. Miner, E. M.; Fukushima, T.; Sheberla, D.; Sun, L.; Surendranath, Y.; Dincă, M., Electrochemical oxygen reduction catalysed by Ni3(hexaiminotriphenylene)2. Nat. Commun. 2016, 7 (1), 10942.

14. Diercks, C. S.; Lin, S.; Kornienko, N.; Kapustin, E. A.; Nichols, E. M.; Zhu, C.; Zhao, Y.; Chang, C. J.; Yaghi, O. M., Reticular Electronic Tuning of Porphyrin Active Sites in Covalent Organic Frameworks for Electrocatalytic Carbon Dioxide Reduction. J. Am. Chem. Soc. 2018, 140 (3), 1116-1122.

15. Qin, J.-S.; Du, D.-Y.; Guan, W.; Bo, X.-J.; Li, Y.-F.; Guo, L.-P.; Su, Z.-M.; Wang, Y.-Y.; Lan, Y.Q.; Zhou, H.-C., Ultrastable Polymolybdate-Based Metal-Organic Frameworks as Highly Active Electrocatalysts for Hydrogen Generation from Water. J. Am. Chem. Soc. 2015, 137 (22), 7169-7177.

16. Duan, J.; Chen, S.; Zhao, C., Ultrathin metal-organic framework array for efficient electrocatalytic water splitting. Nat. Commun. 2017, 8 (1), 15341.

17. Zhao, S.; Wang, Y.; Dong, J.; He, C.-T.; Yin, H.; An, P.; Zhao, K.; Zhang, X.; Gao, C.; Zhang, L.; Lv, J.; Wang, J.; Zhang, J.; Khattak, A. M.; Khan, N. A.; Wei, Z.; Zhang, J.; Liu, S.; Zhao, H.; Tang, Z., Ultrathin metal-organic framework nanosheets for electrocatalytic oxygen evolution. Nat. Energ. 2016, 1 (12), 16184. 
18. Li, F.-L.; Shao, Q.; Huang, X.; Lang, J.-P., Nanoscale Trimetallic Metal-Organic Frameworks Enable Efficient Oxygen Evolution Electrocatalysis. Angew. Chem. Int. Ed. 2018, 57 (7), 1888-1892.

19. Bantignies, J. L.; Deabate, S.; Righi, A.; Rols, S.; Hermet, P.; Sauvajol, J. L.; Henn, F., New Insight into the Vibrational Behavior of Nickel Hydroxide and Oxyhydroxide Using Inelastic Neutron Scattering, Far/Mid-Infrared and Raman Spectroscopies. J. Phys. Chem. C 2008, 112 (6), 2193-2201.

20. Cheng, W.; Zhao, X.; Su, H.; Tang, F.; Che, W.; Zhang, H.; Liu, Q., Lattice-strained metal-organicframework arrays for bifunctional oxygen electrocatalysis. Nat. Energ. 2019, 4 (2), 115-122.

21. Li, J.; Zhang, Y.; Kornienko, N., Heterogeneous electrocatalytic reduction of CO2 promoted by secondary coordination sphere effects. New J. Chem. 2020, 44 (11), 4246-4252.

22. Guo, Y.; Shi, W.; Yang, H.; He, Q.; Zeng, Z.; Ye, J.-y.; He, X.; Huang, R.; Wang, C.; Lin, W., Cooperative Stabilization of the [Pyridinium-CO2-Co] Adduct on a Metal-Organic Layer Enhances Electrocatalytic CO2 Reduction. J. Am. Chem. Soc. 2019, 141 (44), 17875-17883.

23. Wu, H.; Chua, Y. S.; Krungleviciute, V.; Tyagi, M.; Chen, P.; Yildirim, T.; Zhou, W., Unusual and Highly Tunable Missing-Linker Defects in Zirconium Metal-Organic Framework UiO-66 and Their Important Effects on Gas Adsorption. J. Am. Chem. Soc. 2013, 135 (28), 10525-10532.

24. Slater, B.; Wang, Z.; Jiang, S.; Hill, M. R.; Ladewig, B. P., Missing Linker Defects in a Homochiral Metal-Organic Framework: Tuning the Chiral Separation Capacity. J. Am. Chem. Soc. 2017, 139 (50), $18322-18327$.

25. Liu, L.; Chen, Z.; Wang, J.; Zhang, D.; Zhu, Y.; Ling, S.; Huang, K.-W.; Belmabkhout, Y.; Adil, K.; Zhang, Y.; Slater, B.; Eddaoudi, M.; Han, Y., Imaging defects and their evolution in a metal-organic framework at sub-unit-cell resolution. Nat. Chem. 2019, 11 (7), 622-628.

26. Zhang, G.; Fei, H., Missing metal-linker connectivities in a 3-D robust sulfonate-based metalorganic framework for enhanced proton conductivity. Chem. Commun. 2017, 53 (29), 4156-4159.

27. Xue, Z.; Liu, K.; Liu, Q.; Li, Y.; Li, M.; Su, C.-Y.; Ogiwara, N.; Kobayashi, H.; Kitagawa, H.; Liu, M.; Li, G., Missing-linker metal-organic frameworks for oxygen evolution reaction. Nat. Commun. 2019, $10(1), 5048$.

28. van Putten, R.-J.; van der Waal, J. C.; de Jong, E.; Rasrendra, C. B.; Heeres, H. J.; de Vries, J. G., Hydroxymethylfurfural, A Versatile Platform Chemical Made from Renewable Resources. Chemical Reviews 2013, 113 (3), 1499-1597.

29. Li, K.; Sun, Y., Electrocatalytic Upgrading of Biomass-Derived Intermediate Compounds to ValueAdded Products. Chemistry - A European Journal 2018, 24 (69), 18258-18270.

30. Rosi, N. L.; Kim, J.; Eddaoudi, M.; Chen, B.; O'Keeffe, M.; Yaghi, O. M., Rod Packings and Metal-Organic Frameworks Constructed from Rod-Shaped Secondary Building Units. J. Am. Chem. Soc. 2005, 127 (5), 1504-1518.

31. Caskey, S. R.; Wong-Foy, A. G.; Matzger, A. J., Dramatic Tuning of Carbon Dioxide Uptake via Metal Substitution in a Coordination Polymer with Cylindrical Pores. J. Am. Chem. Soc. 2008, 130 (33), 10870-10871.

32. Grant Glover, T.; Peterson, G. W.; Schindler, B. J.; Britt, D.; Yaghi, O., MOF-74 building unit has a direct impact on toxic gas adsorption. Chem. Eng. Sci. 2011, 66 (2), 163-170.

33. Jiang, H.; Wang, Q.; Wang, H.; Chen, Y.; Zhang, M., MOF-74 as an Efficient Catalyst for the LowTemperature Selective Catalytic Reduction of NOx with NH3. ACS App. Mater. Interfaces 2016, 8 (40), 26817-26826.

34. Wang, X. P.; Wu, H. J.; Xi, S. B.; Lee, W. S. V.; Zhang, J.; Wu, Z. H.; Wang, J. O.; Hu, T. D.; Liu, L. M.; Han, Y.; Chee, S. W.; Ning, S. C.; Mirsaidov, U.; Wang, Z. B.; Zhang, Y. W.; Borgna, A.; Wang, J.; Du, Y. H.; Yu, Z. G.; Pennycook, S. J.; Xue, J. M., Strain stabilized nickel hydroxide nanoribbons for efficient water splitting. Energy Environ. Sci. 2020, 13 (1), 229-237.

35. Wang, X.; Xi, S.; Lee, W. S. V.; Huang, P.; Cui, P.; Zhao, L.; Hao, W.; Zhao, X.; Wang, Z.; Wu, H.; Wang, H.; Diao, C.; Borgna, A.; Du, Y.; Yu, Z. G.; Pennycook, S.; Xue, J., Materializing efficient methanol oxidation via electron delocalization in nickel hydroxide nanoribbon. Nat. Commun. 2020, 11 (1), 4647. 
36. Cai, M.; Zhang, Y.; Zhao, Y.; Liu, Q.; Li, Y.; Li, G., Two-dimensional metal-organic framework nanosheets for highly efficient electrocatalytic biomass 5-(hydroxymethyl)furfural (HMF) valorization. $J$. Mater. Chem. A 2020, 8 (39), 20386-20392.

37. Cai, M.; Ding, S.; Gibbons, B.; Yang, X.; Kessinger, M. C.; Morris, A. J., Nickel(ii)-modified covalent-organic framework film for electrocatalytic oxidation of 5-hydroxymethylfurfural (HMF). Chem. Commun. 2020.

38. Villajos, J. A.; Jagorel, N.; Reinsch, S.; Emmerling, F., Increasing Exposed Metal Site Accessibility in a Co-MOF-74 Material With Induced Structure-Defects. 2019, 6 (230).

39. Xiang, W.; Ren, J.; Chen, S.; Shen, C.; Chen, Y.; Zhang, M.; Liu, C.-j., The metal-organic framework UiO-66 with missing-linker defects: A highly active catalyst for carbon dioxide cycloaddition. Appl. Energy 2020, 277, 115560.

40. Cliffe, M. J.; Wan, W.; Zou, X.; Chater, P. A.; Kleppe, A. K.; Tucker, M. G.; Wilhelm, H.; Funnell, N. P.; Coudert, F.-X.; Goodwin, A. L., Correlated defect nanoregions in a metal-organic framework. Nat. Commun. 2014, 5 (1), 4176.

41. Wang, J.; Liu, L.; Chen, C.; Dong, X.; Wang, Q.; Alfilfil, L.; AlAlouni, M. R.; Yao, K.; Huang, J.; Zhang, D.; Han, Y., Engineering effective structural defects of metal-organic frameworks to enhance their catalytic performances. J. Mater. Chem. A 2020, 8 (8), 4464-4472.

42. Hall, D. S.; Lockwood, D. J.; Poirier, S.; Bock, C.; MacDougall, B. R., Raman and Infrared Spectroscopy of $\alpha$ and $\beta$ Phases of Thin Nickel Hydroxide Films Electrochemically Formed on Nickel. $J$. Phys. Chem. A 2012, 116 (25), 6771-6784.

43. Kober, F. P., Infrared Spectroscopic Investigation of Charged Nickel Hydroxide Electrodes. $J$. Electrochem. Soc. 1967, 114 (3), 215.

44. Heidary, N.; Kornienko, N., Operando vibrational spectroscopy for electrochemical biomass valorization. Chem. Commun. 2020, 56 (62), 8726-8734.

45. Halder, M.; Islam, M. M.; Singh, P.; Singha Roy, A.; Islam, S. M.; Sen, K., Sustainable Generation of $\mathrm{Ni}(\mathrm{OH}) 2$ Nanoparticles for the Green Synthesis of 5-Substituted 1H-Tetrazoles: A Competent Turn on Fluorescence Sensing of H2O2. Omega 2018, 3 (7), 8169-8180.

46. Heidary, N.; Kornienko, N., Operando Raman probing of electrocatalytic biomass oxidation on gold nanoparticle surfaces. Chem. Commun. 2019, 55 (80), 11996-11999.

47. Heidary, N.; Kornienko, N., Electrochemical biomass valorization on gold-metal oxide nanoscale heterojunctions enables investigation of both catalyst and reaction dynamics with operando surfaceenhanced Raman spectroscopy. Chem. Sci. 2020, 11 (7), 1798-1806.

48. Tao, H. B.; Xu, Y.; Huang, X.; Chen, J.; Pei, L.; Zhang, J.; Chen, J. G.; Liu, B., A General Method to Probe Oxygen Evolution Intermediates at Operating Conditions. Joule 2019, 3 (6), 1498-1509.

49. Gobal, F.; Valadbeigi, Y.; Kasmaee, L. M., On the significance of hydroxide ion in the electrooxidation of methanol on Ni. J. Electroanal. Chem. 2011, 650 (2), 219-225.

50. Chen, D.; Minteer, S. D., Mechanistic study of nickel based catalysts for oxygen evolution and methanol oxidation in alkaline medium. J. Power Sources 2015, 284, 27-37. 


\section{Supporting Information for:}

Rational incorporation of missing linker defects within metal-organic frameworks generates highly active electrocatalytic sites

Nina Heidary ${ }^{1}$, Daniel Chartrand ${ }^{1}$, and Nikolay Kornienko ${ }^{1 *}$

${ }^{1}$ Department of Chemistry, Université de Montréal, 1375 Avenue Thérèse-Lavoie-Roux, Montréal, QC H2V 0B3, Canada.

*E-mail: nikolay.kornienko@ umontreal.ca 
MOF Synthesis: The MOFs were synthesized in a one-pot solution phase method. $20 \mathrm{~mL}$ scintillation vials were filled with $10 \mathrm{~mL}$ of DMF, $0.5 \mathrm{~mL}$ of water, $136.5 \mathrm{mg}$ of nickel nitrate hexahydrate, and $52 \mathrm{mg}$ of 2,5-dihydroxyterepthalic acid. For Ni74D, $39 \mathrm{mg}$ 2,5-dihydroxyterepthalic acid and $13 \mathrm{mg} 2$ -

hydroxyterepthalic acid were used. The solutions were briefly sonicated to dissolve all of the components and placed into an oven at $100^{\circ} \mathrm{C}$ for 24 hrs. Following the MOF growth, the residual solvent was removed by soaking in methanol at $70^{\circ} \mathrm{C}$ for 5 days while periodically refreshing the methanol and finally vacuum drying at $80^{\circ} \mathrm{C}$ for 3 days.

Physical Characterization: XRD spectra were taken on the MOF powders both after synthesis and after electrochemical measurements in which the MOF electrodes were simply rinsed with water and allowed to dry in an air environment. For SEM measurements, the MOFs were dispersed in ethanol and drop cast onto titanium foil or the MOF electrodes (described below) were imaged after simply rinsing with water.

\section{Infrared Spectroscopy:}

A ThermoFischer Nicolet 380 FTIR-ATR was used for all IR measurements. The MOFs, either dry or dispersed in $\mathrm{KOH}$ or HMF were pressed directly onto a diamond coated ZnSe ATR waveguide. An atmospheric background spectrum was taken before every MOF spectrum. Each spectrum was recorded with an accumulation of 400 scans with a resolution of $4 \mathrm{~cm}^{-1}$. OPUS 7.0 software was used to carefully subtract out the air and $\mathrm{KOH}$ background spectra. This software was also used for baseline correction and peak fitting.

Electrochemistry: For all electrochemical experiments a Biologic VMP 200 potentiostat, coupled with EC-lab software was utilized. Custom-built 2-compartment glass electrochemical cells were used. $\mathrm{Ag} / \mathrm{AgCl}$ reference electrodes and graphite rod counter electrodes were employed in electrochemical measurements. Periodically, the $\mathrm{Ag} / \mathrm{AgCl}$ reference electrode was checked vs. a master electrode to ensure that the potential did not significantly drift and affect the measurements. $10 \mathrm{mM} \mathrm{KOH}$ with $1 \mathrm{M}$ $\mathrm{KCl}$ supporting electrolyte was used for all measurements. The solution resistance was compensated for at $85 \%$ with the ZIR function in EC-Lab, which measures this value at open circuit potentials at $100 \mathrm{KHz}$ frequency.

To fabricate working electrodes, first a catalyst ink was made from $150 \mu \mathrm{L}$ water, $450 \mu \mathrm{L}$ ethanol, $5 \mu \mathrm{L}$ nafion solution (5\% wt. in water), $6 \mathrm{mg}$ MOF, and approx. $0.1 \mathrm{mg}$ multiwall carbon nanotubes (40 nm diameter). This solution was sonicated for 20 minutes to render it homogeneously dispersed and was subsequently drop cast onto either toray caron paper or onto the glassy carbon rotating disk electrode surface and let dry in ambient conditions for 20 minutes prior to use. Product quantification was performed with NMR measurements with an acetate internal standard and calibration curves performed with known concentrations of standards. 


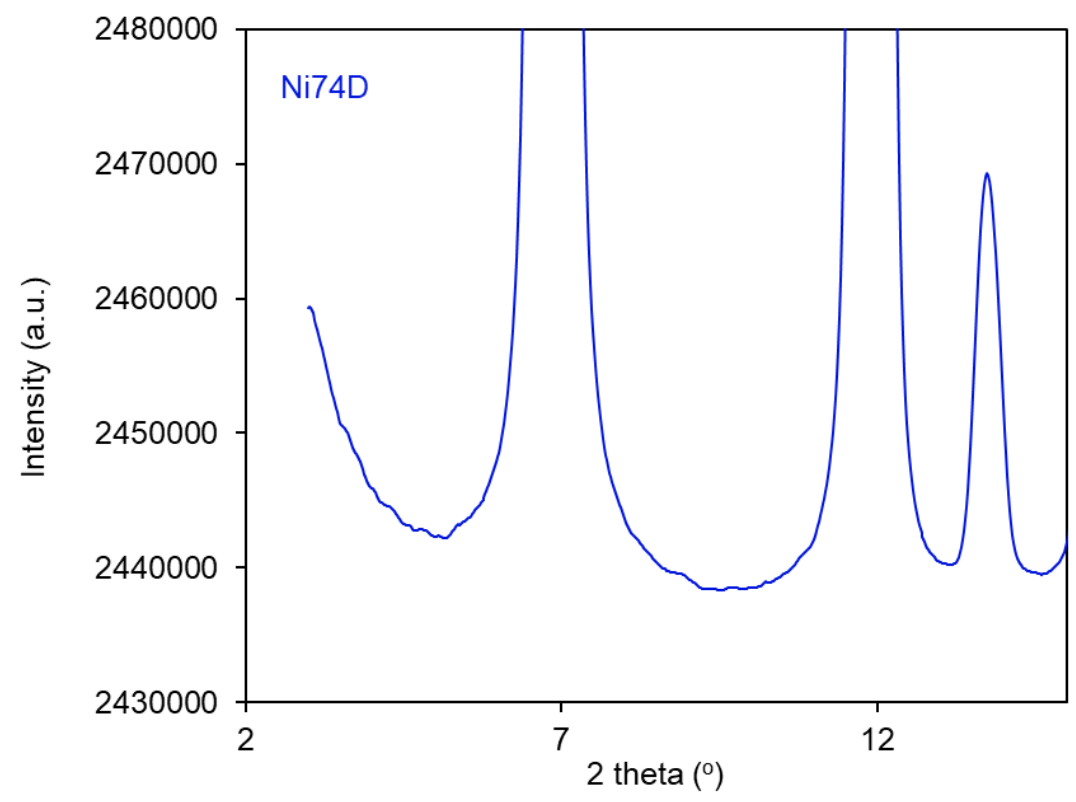

Figure S1: XRD spectrum of Ni74D zoomed in shows a lack of additional peaks at low wavenumbers and therefore no evidence for ordering of the defects. 


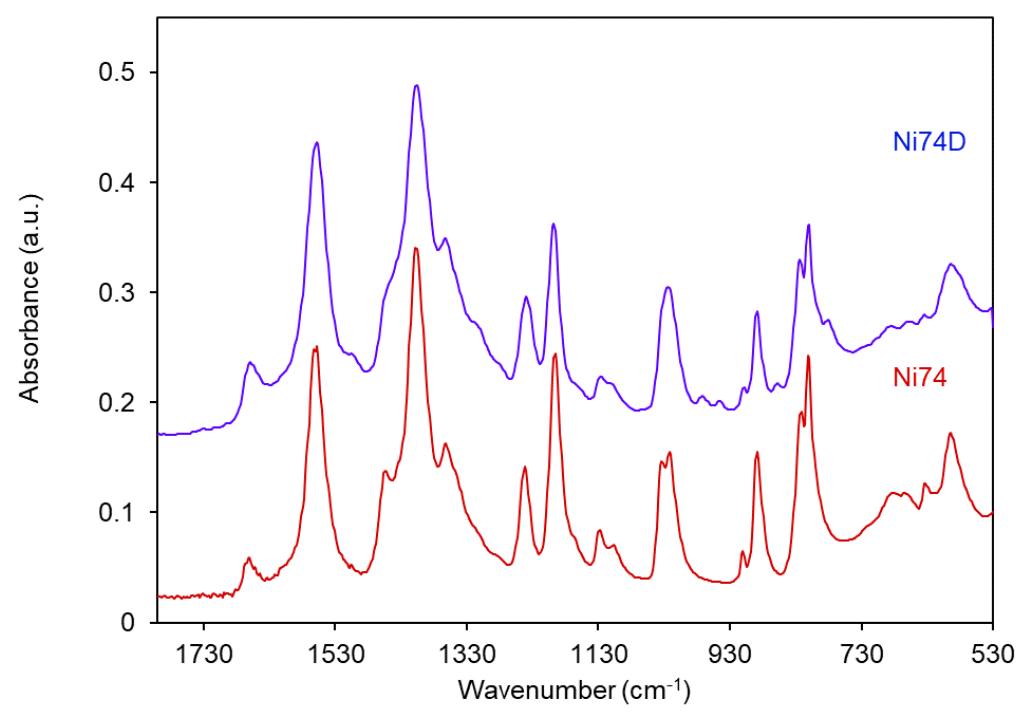

Figure S2: IR spectra of dry Ni74 and Ni74D MOFs show a peak broadening of Ni74D, consistent with a decrease in crystallinity. 

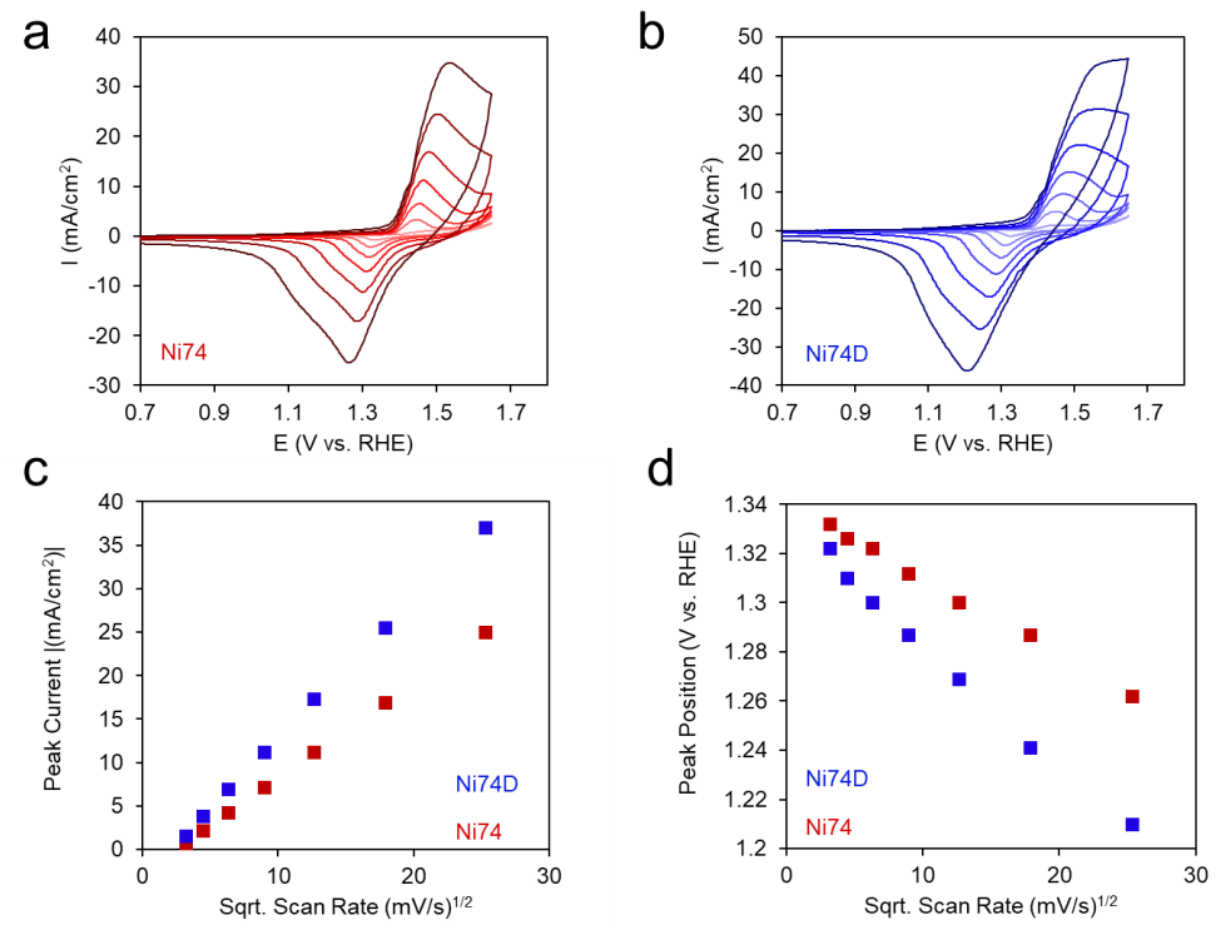

Figure S3: CVs of Ni74 (a) and Ni74D (b) taken at different scan rates. The peak current scales with the square root of the scan rate, indicative of diffusion-limited charge transport (c). The peak position similarly shifts with scan rate (d). 


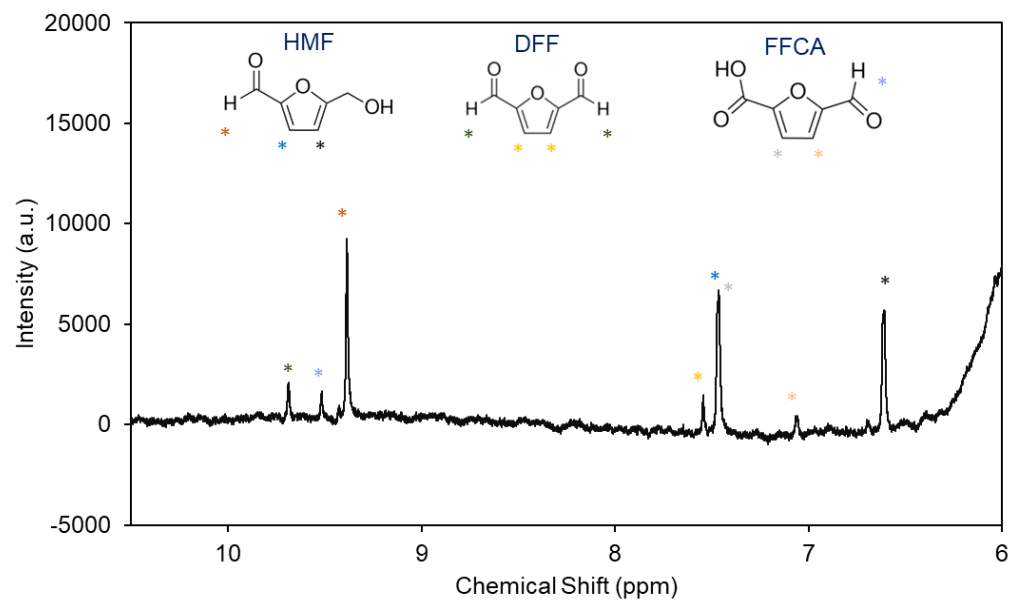

Figure S4: NMR spectra of HMF oxidation of Ni74D taken at 2 hrs reveals the formation of DFF and FFCA 
a

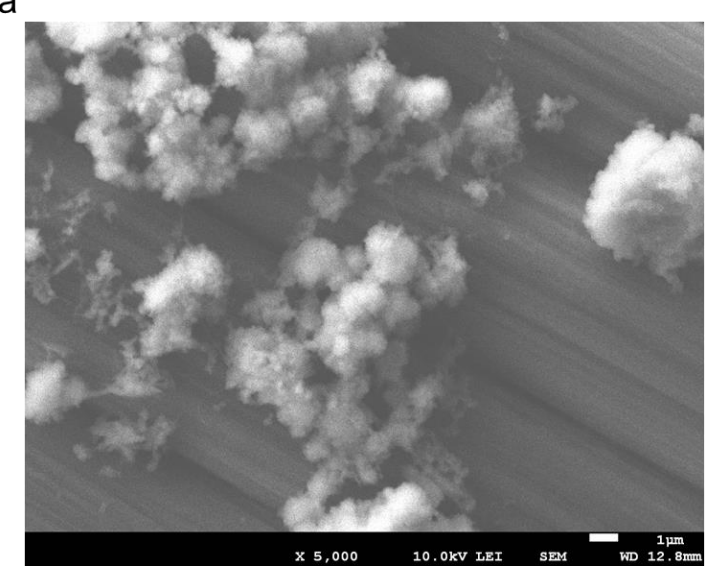

b

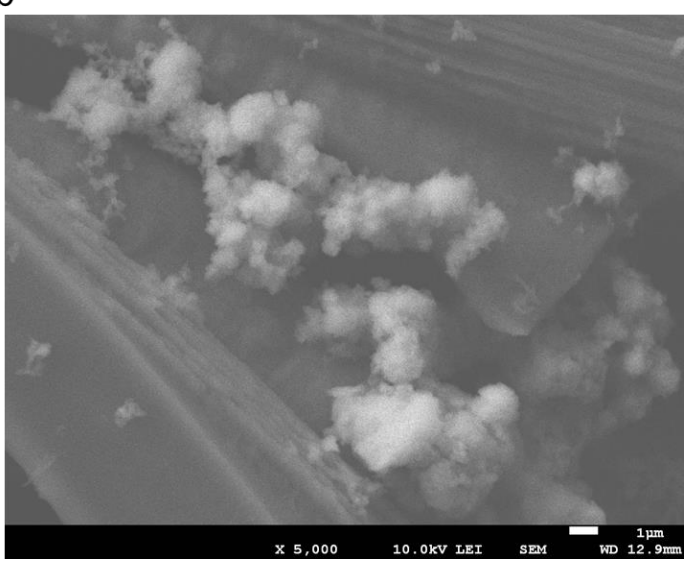

Figure S5: Representative SEM images of Ni74D before (a) and after (b) electrolysis for 24 hrs. 


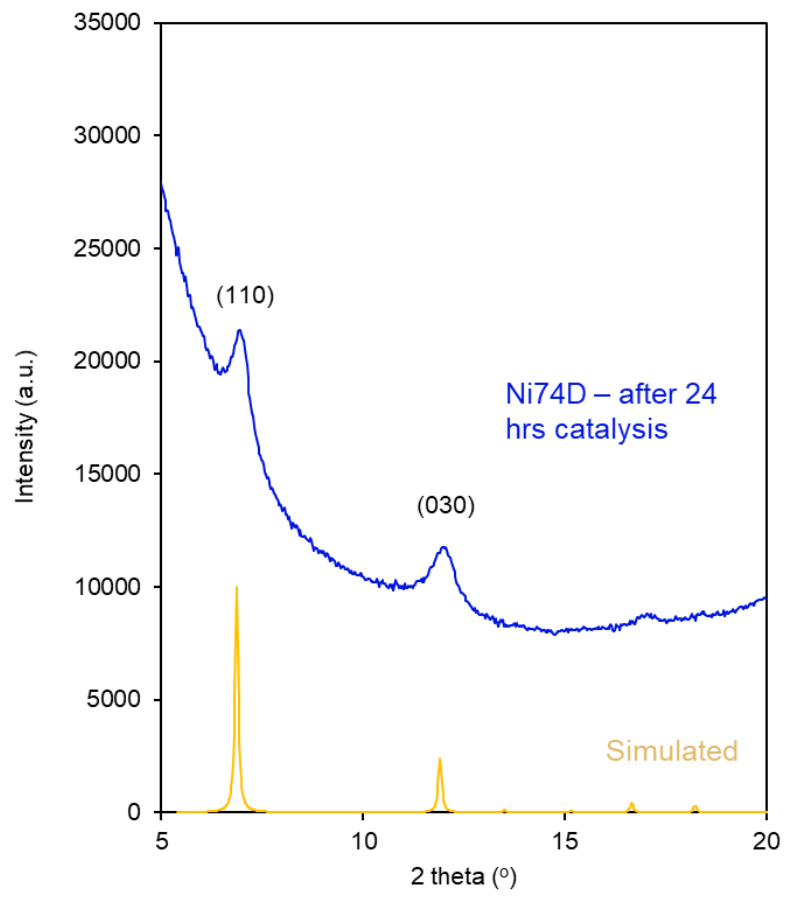

Figure S6: XRD spectrum of Ni74D after 24 hrs of electrolysis show that the principal (110) and (030) peaks remain. 
a

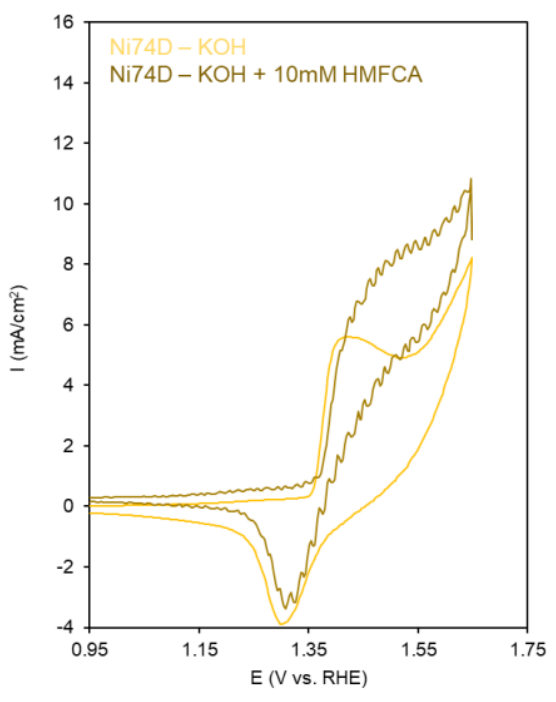

b

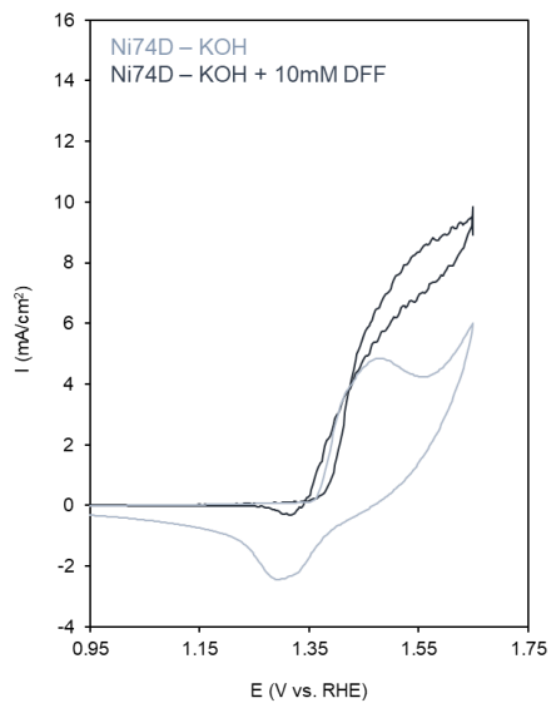

Figure S7: CVs of HMFCA oxidation (a) and DFF oxidation (b) with Ni74D. 